\title{
Parallel grafts and physician modified endografts for endovascular repair of the aortic arch
}

\author{
Marvin D. Atkins, Alan B. Lumsden \\ Houston Methodist Hospital and Houston Methodist DeBakey Cardiovascular Surgery Associates, Houston, TX, USA \\ Correspondence to: Marvin D. Atkins, MD, FACS. Associate Professor of Surgery, Department of Cardiovascular Surgery, Houston Methodist \\ Hospital, 6550 Fannin Street, Smith Tower 1401, Houston, TX 77030, USA. Email: mdatkins@houstonmethodist.org.
}

\begin{abstract}
Aortic arch aneurysms are a challenging clinical problem especially in high-risk patients. Open aortic arch replacement, even in the best of centers, carries significant risk of stroke or death in this high-risk population. Many high-risk patients are deemed inoperable and not offered repair. Branched and fenestrated thoracic endografts are currently undergoing clinical trials in the United States but are not yet commercially available. Many elderly and frail patients have significant brachiocephalic occlusive disease or anatomy excluding them for consideration for such clinical trials. These patients also present with acute aortic syndromes requiring urgent or emergent repair and are unable to participate in clinical trials due to the time required to have such devices available. Alternative endovascular therapies, including parallel stent grafts (including Chimneys, Snorkels and Periscopes) and physician modified thoracic endografts, have been used to treat such high-risk patients combined with commercially available thoracic endovascular aneurysm repair (TEVAR) devices. This paper aims to review the techniques and current reported outcomes from parallel stent grafts and physician modified devices used to treat high risk patients undergoing repair for aortic arch pathologies.
\end{abstract}

Keywords: Parallel grafts; physician modified endoluminal graft; chimney; snorkel; periscope; in-situ fenestration

Submitted Sep 17, 2021. Accepted for publication Oct 27, 2021.

doi: $10.21037 /$ acs-2021-taes-171

View this article at: https://dx.doi.org/10.21037/acs-2021-taes-171

\section{Introduction}

Aortic arch aneurysms have been a formidable and challenging clinical problem since the first attempts at open surgical repair in 1957 by Drs. Denton Cooley and Michael DeBakey at our institution (1). The early experience with the management of aortic arch aneurysms was complicated by exceedingly high rates of stroke, major bleeding and death, with surgical treatment limited to just a few high volume centers around the world. Over the last six decades, advances in cardiopulmonary bypass, myocardial protection, cardiovascular anesthetic techniques, the use of deep hypothermic circulatory arrest, and antegrade and/or retrograde cerebral perfusion have significantly improved the outcomes of open aortic arch aneurysm surgery. Svensson et al. from the Cleveland Clinic have reported their outcomes in highly selected patients undergoing elective aortic arch surgery with 2\% mortality and 2\% stroke rate (2). Shah et al. examined their outcomes of ascending and transverse aortic arch surgery for both aneurysms and aortic dissection in octogenarians (37 patients/4.8\%) compared to younger patients (742 patients/95.2\%), and found acceptable rates of stroke (8\%) and death $(13.5 \%)$ in octogenarians that was not statistically different from the younger cohort (3). Open surgical repair is still considered the standard of care for aortic arch aneurysms, however, morbidity and mortality remains significant with reported rates ranging between $3.7 \%$ and $14 \%$ for mortality and $4 \%$ to $10 \%$ for neurological events (4-9).

It is difficult to compare aortic arch surgery to techniques of endovascular repair in the current literature due to the inherent bias in patient selection. Truly high-risk patients are more likely to undergo either non-operative repair or 
endovascular repair, including the techniques of parallel grafting or physician modified grafts. Patients undergoing open surgical repair, even those with advanced age, have passed clinicians' "eyeball test" and are appropriately offered open repair. Randomized trials of open aortic arch repair versus endovascular repair are unlikely as the current protocols for trials of branched and fenestrated thoracic endovascular devices have enrolled patients that are deemed "high-risk" by clinicians. We will not see comparable studies of parallel stent grafts or physician modified grafts as these are typically regarded as bail out procedures or are used in the setting of patients who are not candidates for clinical trial devices. The current evidence for the use of parallel grafts or physician modified grafts is based upon small, typically single center, case series and a limited number of multi-institutional collaborations, making direct comparisons to open arch repair impossible.

\section{Hybrid aortic arch repair}

Thoracic endovascular aneurysm repair (TEVAR) has supplanted open surgical repair as the current standard of care for pathologies involving the descending thoracic aorta with associated decreased risk of morbidity, stroke, spinal cord ischemia, and mortality. Successful thoracic aortic aneurysm repair is predicated upon adequate proximal and distal seal zones to avoid endoleak and device migration. It is generally accepted that a seal zone of at least $2.5 \mathrm{~cm}$ is necessary for seal in fusiform thoracic aneurysms. In patients without an adequate proximal landing zone, a combination of open and endovascular surgery (hybrid aortic arch repair) can create an adequate proximal seal zone to allow for TEVAR. Extra-anatomic bypasses including carotid-carotid with carotid-left-subclavian bypass can extend the area of seal zone proximally to allow successful TEVAR of a distal arch aneurysm. In patients whose aneurysm extends more proximally and would require coverage of the innominate artery, a more proximal debranching procedure is required. Bavaria et al. (10) described a classification system for total arch debranching via median sternotomy (Figure 1). Type 1 repair involves placement of a side biting clamp on the ascending aorta, without the need for cardiopulmonary bypass and debranching of the brachiocephalic vessels. The stent graft repair lands in the native ascending aorta above the takeoff of the bypass grafts. Type 2 repair involves cardiopulmonary bypass and replacement of the ascending aorta with a graft to create an area for stent graft seal. The brachiocephalic branches are bypassed from grafts arising from the ascending graft similar to type 1 repair. The TEVAR lands in the graft material of the new ascending aorta. Type 3 repair involves conventional arch replacement under hypothermic circulatory arrest and supra-aortic debranching. TEVAR can be performed in the same setting (frozen elephant trunk) or as a secondary procedure. Zone 2 arch replacement has also been proposed as another type of hybrid procedure that allows for debranching of the innominate and left common carotid artery with the distal anastomosis in zone 2 between the left common carotid and left subclavian (Figure 2). The purported benefits of this operation are that the distal anastomosis is technically easier as opposed to an anastomosis deep in the chest, and the left recurrent laryngeal nerve is typically avoided. If the patient requires further coverage of the aorta then a TEVAR device can cover the left subclavian, with or without left carotid subclavian bypass in the neck. Thoracic branched endografts have also been used in this setting with the branched endograft in the left subclavian artery.

Elhelali et al. reviewed the available literature through March 2021 for the Cochrane database of systematic reviews, including any randomized controlled trials (RCT) or controlled clinical trials (CCT) comparing hybrid repair to open surgical repair for thoracic aortic arch aneurysms (11). Their extensive literature review of 1,959 reports of hybrid arch repair did not find a single study that met the inclusion criteria. Their inclusion criteria were guided by the International Aortic Arch Surgery Study Group for defining primary and secondary outcomes (12). Primary outcomes included aneurysm related mortality at thirty days and twelve months, any neurological deficit (stroke, paraplegia), or cardiopulmonary complications. Secondary outcomes included graft patency and reintervention rate. The reviewed studies were excluded for various reasons, most commonly because they were retrospective reviews without a comparator rather than RCT or CCT. The authors of this Cochrane review concluded that there is insufficient evidence to determine the safety and effectiveness of hybrid repair compared to open surgical repair in patients with thoracic aortic arch aneurysms. High quality RCTs or CCTs are necessary to determine the role of hybrid repair in the management of thoracic aortic arch aneurysms. Hybrid aortic arch repair creates an area for proximal seal where one does not exist. This procedure requires sternotomy and possibly cardiopulmonary bypass depending upon the area for proximal seal. Many patients who are elderly or high-risk are not candidates for hybrid operations due to their frailty 


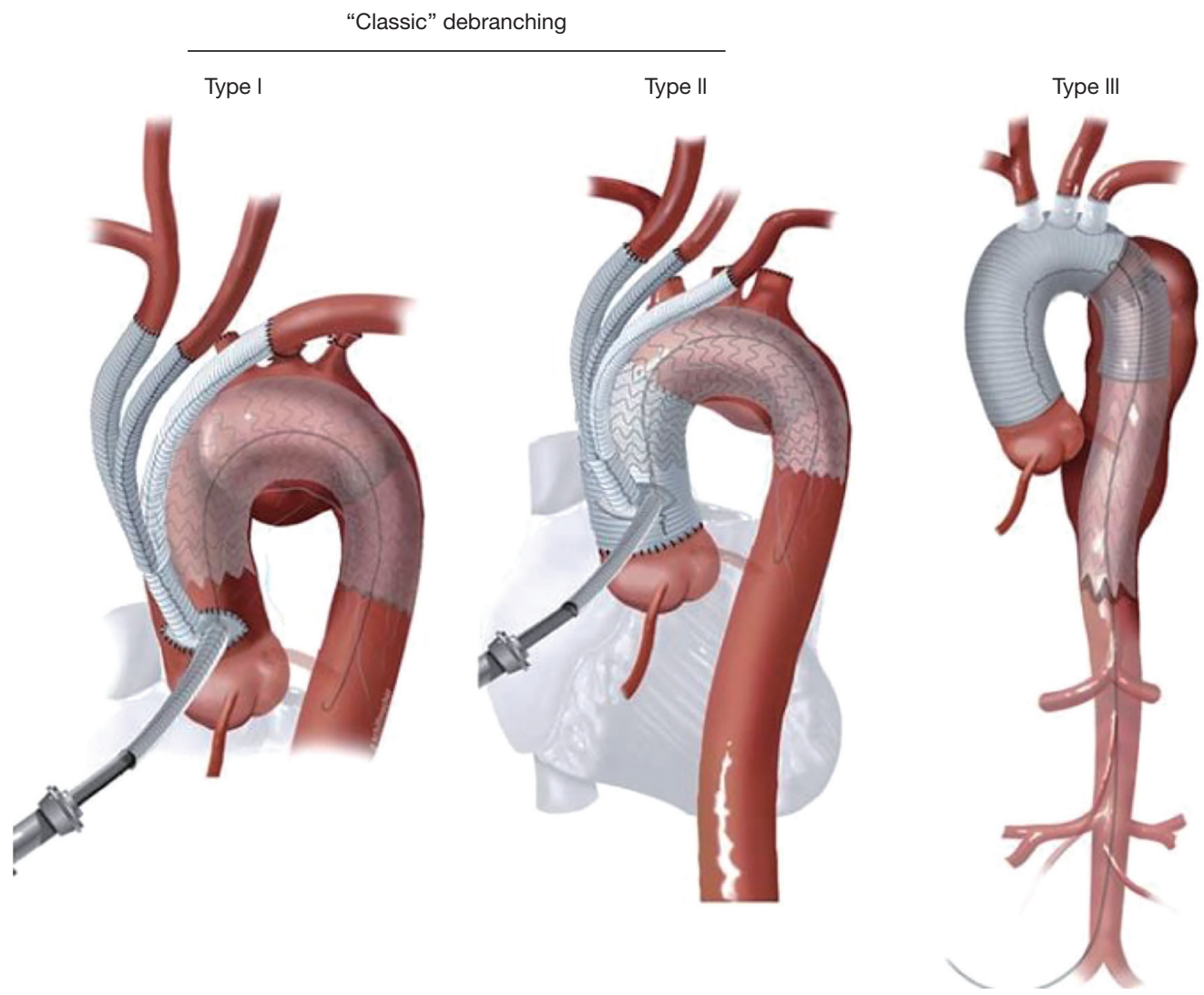

Figure 1 Bavaria hybrid arch repair. University of Pennsylvania classification system for hybrid aortic arch repair.
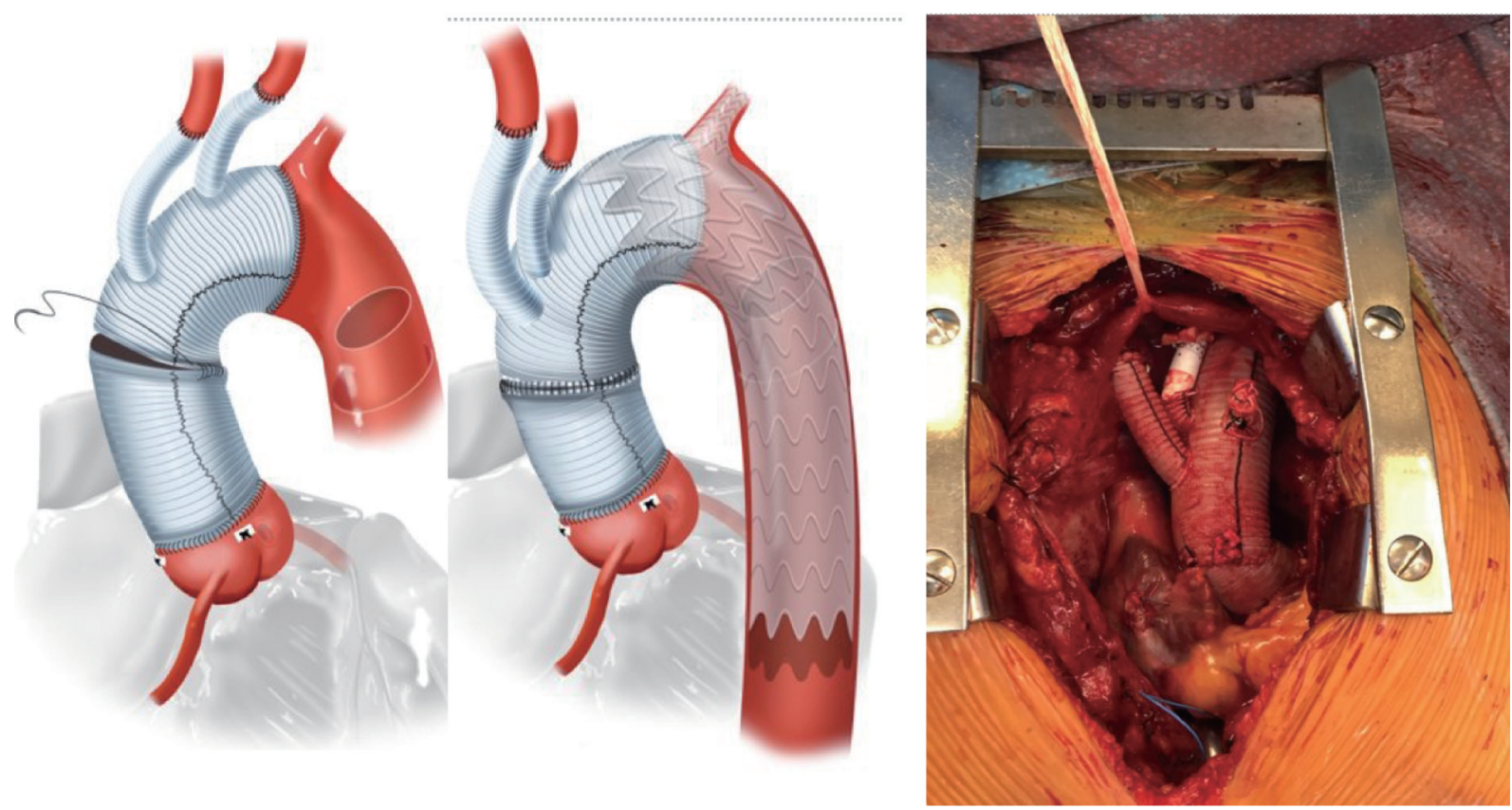

Figure 2 Zone 2 arch repair. Zone 2 aortic arch repair with debranching of the innominate artery and left common carotid artery. 


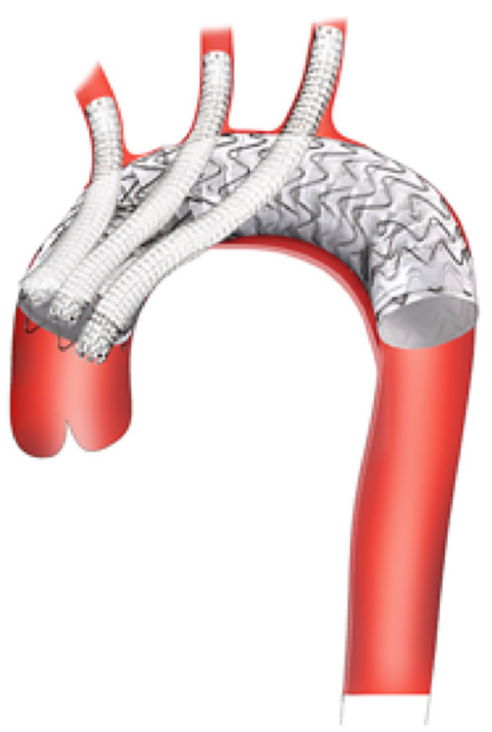

Figure 3 Chimney grafts. Arch repair with placement of parallel grafts in all three brachiocephalic vessels. The parallel grafts should extend proximal to the TEVAR graft. Multiple parallel grafts significantly increase the risk of Type 1 endoleak and generally should be avoided if possible. TEVAR, thoracic endovascular aneurysm repair.

or comorbidities. In such patients, alternative endovascular strategies, as discussed below, may be the only option.

\section{Parallel grafts}

Fenestrated and branched endografts are currently undergoing clinical trials in the United States and at present there are no commercially available devices for use in the aortic arch. Many of these devices are custommade and require several months to manufacture prior to implantation. Such custom devices are typically only available as part of investigational device exemption (IDE) programs, which are not widely available. For patients requiring urgent/emergent repair, or when there is inadvertent coverage of a supra-aortic branch vessel, an immediate bail out option is required. Parallel stent grafting on an emergent basis was the first such bail out maneuver used in this setting.

Parallel stent grafting is a term encompassing multiple techniques where branch vessel stent grafts run parallel to a main body aortic graft. Other terms include Chimneys, Periscopes, Snorkels or Sandwich technique depending upon the orientation of the branch grafts (Figure 3). Parallel

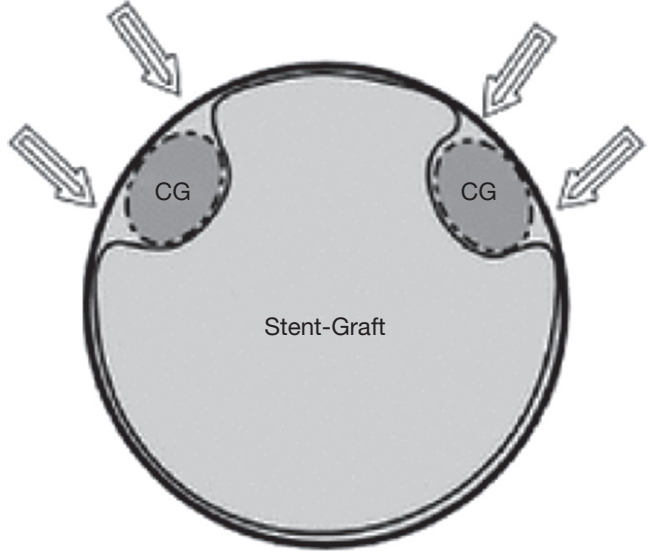

Figure 4 Parallel graft gutters. Parallel grafts create gutters between the aortic wall and the stent graft and may lead to Type 1 endoleak.

grafting is technically easier than in situ fenestration or physician modification of a stent graft device. In the sometimes-litigious world of surgical practice, parallel grafting can be an attractive alternative compared to modification of commercially available devices and the unknown long term structural effects of those modifications.

Parallel stent grafting in the aortic arch carries similar concerns for complications compared to open surgical repair including stroke, need for secondary interventions, and operative mortality. What is unique with parallel stent grafting is the planned disruption of the proximal seal zone of a TEVAR device. Creation of such gutters between the TEVAR device, the parallel stent graft and the aortic wall are the Achilles heel of this technique. These gutters represent Type $1 \mathrm{~A}$ or $1 \mathrm{~B}$ endoleaks that if persistent can lead to systemic arterial pressure within the aneurysm sac increasing the risk of aneurysm rupture. The risk of Type 1 endoleak is reported to be as high as $23 \%$ in midterm follow-up (13) (Figure 4).

Lindblad et al. reported a meta-analysis of parallel grafts in abdominal and thoracic aortic stent grafting and included a review of those involving the aortic arch (14). They found 17 series and 23 case reports involving 314 patients who underwent parallel grafting of the supra-aortic trunks. Ninety-eight of the reported cases were in emergent situations. Early parallel graft patency was $99 \%$ with endoleak reported in 33 patients (11\%). Thirty-day mortality occurred in 14 patients (4\%) and procedure related complications were reported in 20 patients $(6 \%)$. 
Fifteen (45\%) thoracic arch Type 1 endoleaks were observed and seven were reported to seal. Nine $(27 \%)$ thoracic endoleaks were embolized or glued and five were treated by extension of the thoracic endograft. Three thoracic cases required open conversion, all for Type 1 endoleak.

Voskresensky et al. (15) from the University of Florida reported their single center aortic arch chimney stent experience in 27 patients deemed unfit for open repair between 2002 and 2015. One third of patients were either symptomatic $(26 \%)$ or ruptured $(7 \%)$ at the time of presentation; $41 \%$ had previous open or endovascular repair of the arch or descending thoracic aorta. Chimney stents were planned in $75 \%$ of cases with the remainder placed for inadvertent coverage or encroachment on a brachiocephalic vessel. Five patients (19\%) had two or more chimney grafts placed. Overall technical success was $89 \%$ (one intraoperative death, two persistent Type 1A endoleak on subsequent imaging). Thirty-day mortality was low at $4 \%$ with one intraoperative death of a ruptured arch aneurysm. Seven patients (26\%) experienced a major complication (stroke, three; respiratory failure, three; death, one). Nine patients $(33 \%)$ required reintervention and no chimney occlusions occurred in the mid-term follow up. In this group of high-risk patients, the authors concluded that parallel stent grafting can be safely performed with high technical success and acceptable mid-term outcomes. Given the high rate of reintervention with parallel grafting, the authors stressed the need for diligent follow up.

\section{Endovascular technique of parallel grafts}

The technique of parallel grafting is relatively straight forward albeit with a few caveats. It is universally accepted that parallel grafts should be covered stent grafts as opposed to bare metal stents to enhance sealing. In the parallel graft technique, excess oversizing of the main body aortic endograft is needed to facilitate formation of channels lateral to the stent graft in order to accommodate and hopefully mold around the parallel graft. An undersized main body graft leads to the formation of gutters and Type $1 \mathrm{~A}$ or $1 \mathrm{~B}$ Endoleak. It is important to have the parallel grafts extend beyond the main body stent graft for at least $1-2 \mathrm{~cm}$. Many interventionalists will reinforce the parallel grafts with self-expanding or even balloon expandable stents to prevent crushing of the parallel graft from the radial force of the aortic grafts. In our practice we tend to reinforce the origins of the brachiocephalic vessels inside of the parallel grafts with a self-expandable stent. The longer a gutter is, the more likely it is to seal along its length if an endoleak occurs. Multiple parallel grafts significantly increase the risk of gutters and endoleak and generally should be avoided if possible.

\section{Physician modified endografts (PMEGs)}

\section{In-situ fenestration}

In-situ arch fenestration has been used by some as a means of revascularizing supra-aortic branch vessels without the need for custom branched or fenestrated grafts or the use of parallel grafts that may compromise the seal zones with the creation of gutters and the risk of endoleak. McWilliams et al. described the first needle in situ fenestration of the left subclavian artery during TEVAR (16). A needle was used to puncture the fabric and serial cutting balloons were used to enlarge the opening. This was followed by covered stent placement through the fenestration into the left subclavian artery. Due to concerns with creating uncontrolled tears in the fabric and Type 3 endoleak, others have used laser or radiofrequency energy to create fenestrations in the TEVAR fabric. Murphy et al. used laser fenestration in 2009 to revascularize the left subclavian artery in a traumatic aortic transection with good results (17). Panneton et al. have one of the largest single center series on laser fenestration and have reported their experience in various aortic pathologies, as well as studying laser fenestration in the laboratory to better define its place in a surgeon's armamentarium (18-20). Laser fenestration has typically been used in urgent or emergent settings as a means to revascularize aortic arch vessels when trial branched or fenestrated devices are not an option or in the setting of unintended coverage of a supraaortic branch vessel (Figure 5).

Anatomical limitations to in-situ fenestration include steep angulation (<30 degrees) of supra-aortic branch vessels or if they arise very anterior or posterior on the arch. Such angulation makes it difficult to align the laser perpendicular to the graft material. Stent graft branches arising at a steep angle, rather than perpendicular to the TEVAR graft, are more prone to develop stenosis or thrombose. Other anatomical limitations to in situ fenestration involve characteristics of the branch vessel being stented. Large vessel diameter $(>10 \mathrm{~mm})$, dissection in the branch vessel, or a short distance to secondary branches (such as the vertebral artery on the left, or the innominate bifurcation on the right) can complicate in situ fenestration.

Riga et al. examined in-situ needle fenestration (21) 

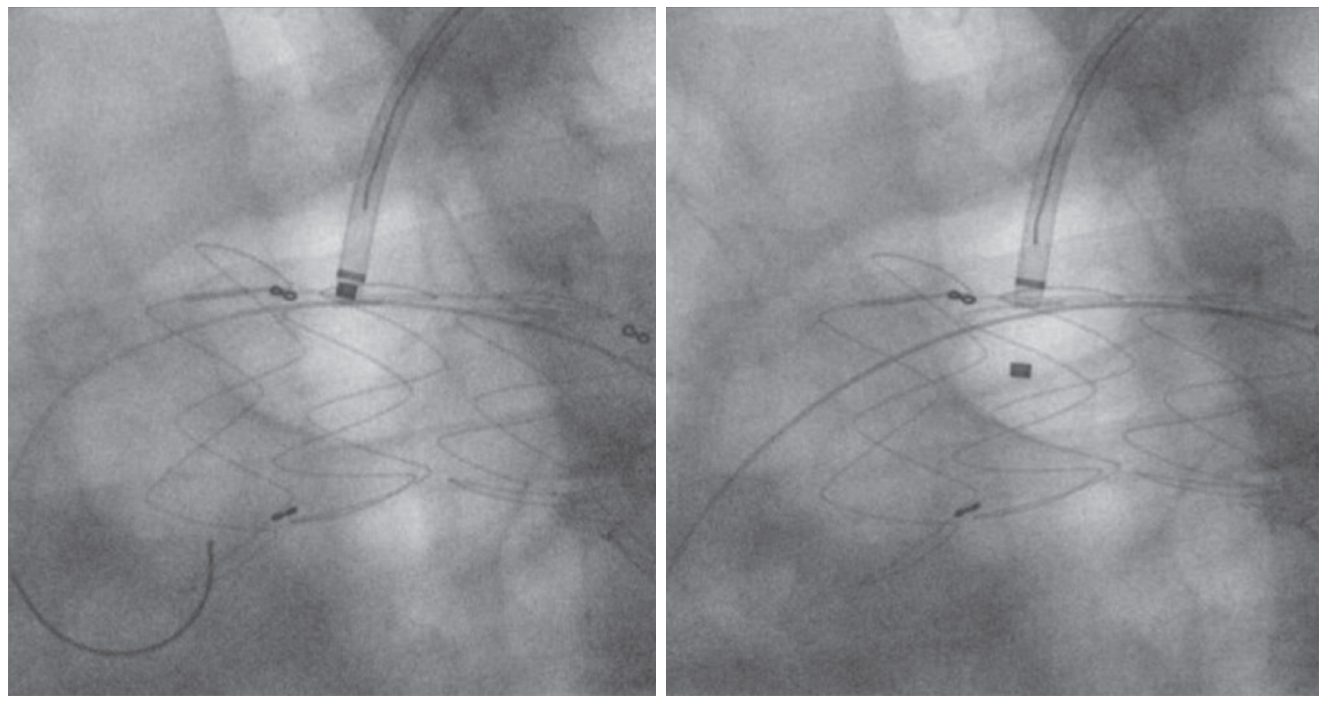

Figure 5 Laser fenestration. Using energy from a laser, a small hole is burned in the fabric of the TEVAR graft and a wire is passed into the lumen. The in-situ fenestration is then balloon dilated, and a covered stent graft is placed with the proximal end flared. TEVAR, thoracic endovascular aneurysm repair.

in a benchtop model utilizing various commercially available TEVAR grafts and found distinct differences among polytetrafluoroethylene (PTFE) and various types of polyester grafts. PTFE was easier to penetrate than polyester but resulted in elliptical or slit like fenestrations. Monofilament twill woven polyester [Medtronic Talent Endograft (Medtronic Vascular, Santa Rosa, CA, USA)] achieved a circular fenestration but not the multifilament, tubular woven polyester of the Cook Zenith Endograft (Cook Inc., Bloomington, IN, USA). The quality of the fenestrations also decreased with the angle of puncture in all materials and cutting balloons created more tears as opposed to standard balloon angioplasty. Panneton et al. have also performed in vitro testing of laser and needle fenestration and found that laser fenestration created more predictable openings with less tears. They also noted more tears of the fabric when dilated to 10 and $12 \mathrm{~mm}$ as compared to $8 \mathrm{~mm}$ balloons. Panneton et al. also caution the use of laser fenestration in the setting of PTFE grafts due to concerns for the intravascular release of hydrogen chloride, trifluoroacetate, and other toxic substances (22). In clinical practice, their group has limited laser fenestration to polyester grafts. Zeng et al. recently reported their in vitro comparison of needle and laser fenestration using various stent grafts and noted that following balloon angioplasty of the fenestrations, there was no significant difference in the quality of the fenestrations between either technique $(23,24)$.

\section{Technique}

Needle or laser fenestration begins with placement of a 7 or $8 \mathrm{Fr}$ sheath in the planned target vessel for revascularization. The size of the sheath is dependent on the size of the planned covered stent graft rather than the diameter of the laser or needle. Typically, an angled Ansel sheath (Cook Medical, Bloomington, IN, USA) or another preformed angled tip allows directionality of the sheath. One can also use one of the newer steerable sheaths to allow maneuvering of the sheath to orient perpendicular to the TEVAR graft. Current options include the Morph AccessPro 6 Fr (BioCardia, Inc., San Carlos, CA, USA) or the TourGuide 6.5, 7, 8.5 Fr (Medtronic Vascular, Santa Rosa, CA, USA). Our preference has been to use a Spectranetics (now Philips) Turbo Elite $2 \mathrm{~mm}$ laser (6 Fr compatible, over an 0.018 guidewire) via a steerable delivery catheter. The laser is oriented perpendicular to the stent graft, and this is confirmed in both a left anterior oblique (LAO) projection as well as a right anterior oblique (RAO) projection to ensure optimal placement perpendicular to the fabric and in the center of the endograft. Standard laser energy is applied using gentle forward pressure on the endograft until the laser visibly penetrates the fabric. The 0.018 wire within the laser is now advanced, the laser is removed and a secondary catheter [we prefer a QuickCross (Philips, Cambridge, MA, USA)] is advanced to exchange it with a stiff 0.035 wire. Balloon angioplasty 
of the fenestration is performed with a $6 \mathrm{~mm}$ angioplasty balloon. The fenestration is now stented with an $8-10 \mathrm{~mm}$ iCast balloon expandable covered stent (Getinge, Wayne, NJ, USA) or Gore VBX balloon expandable stent (Gore, Flagstaff, AZ, USA). One quarter of the stent graft is within the TEVAR and the other $3 / 4$ is within the branch vessel. The intragraft portion of the balloon expandable stent graft is flared with a $14 \times 20$ balloon. This balloon angioplasty must be outside of the fenestration in order to prevent tears of the TEVAR fabric. The current sizes of balloon expandable stent grafts make in situ fenestration possible for most left subclavian and left common carotid arteries. The innominate artery is often outside of the size range for laser fenestration, especially in the setting of a bovine trunk, and this limits the technique's use for total endovascular arch coverage.

\section{Outcomes of in-situ fenestration}

Panneton et al. reported single center results of 36 patients undergoing attempted in situ laser fenestration, which was technically successful in 35/36 cases (97.2\%). Of note, 33 of 35 cases were of laser fenestration of the left subclavian artery alone. Mortality was reported in $5.7 \%$ and stroke in $2.9 \%$. Two patients had minor vascular complications requiring evacuation of arm brachial artery associated hematomas. There were no major fenestration related complications.

Crawford et al. performed a meta-analysis in 2016 of all available series and case reports of in situ fenestration of the aortic arch (25). Sixteen clinical papers (including two case series and 14 case reports) reported in situ fenestration of 46 aortic arch vessels in 44 patients. There were 42 retrograde fenestrations and four antegrade instances of in situ fenestration. The left subclavian was the target vessel in $72 \%$ and the most frequent indication was degenerative aneurysm (43\%). Technical success was high at $96 \%$ and the combined perioperative rate of stroke, paralysis or death was $7 \%$. Most fenestrations were performed in polyester grafts (91\%) as opposed to PTFE (9\%). Short to moderate term reported results were deemed to be acceptable and longer follow up is required due to concerns for durability and the risk of structural integrity of the fenestration/stent graft junction in a mobile aortic arch.

The AARCHIF (Aortic ARCH In situ Fenestration) registry reported the international multicenter experience of in-situ fenestration for aortic arch aneurysms or localized Type A dissections involving Ishimura Zone 0 or 1 pathology. Isolated in situ fenestration of the left subclavian artery was excluded from this analysis (26). Twentyfive patients were treated at nine different institutions in seven different countries between June 2009 and March 2017. Fenestrations were created by either laser, needle or radiofrequency catheters with implantation of covered stent grafts. Unique to this series was fenestration of the brachiocephalic trunk (20 patients), left common carotid (21 patients) and left subclavian (nine patients). Most of the patients in this registry had intraoperative cerebral perfusion during the AARCHIF procedure using extracorporeal circulation with pump driven bypass to both carotid arteries or an extraanatomical femoral-carotid or axillary carotid bypass for cerebral perfusion. Technical success was $94 \%$ (47/50). Mortality occurred in one patient (4\%) with stroke rates of $12 \%$ (three patients). One proximal stent graft non-alignment and four Type III endoleaks (two early, two late) were observed and required reintervention. Mid-term follow up was 31.5 months and showed no late aneurysm related mortality. Late reinterventions occurred in six $(26.1 \%)$ due to Type III endoleak or distal aneurysm expansion requiring intervention.

In situ fenestration, using laser or needle technique, appears to be a reasonable option in the short and possibly mid-term in patients who are not candidates for open/ hybrid aortic repair and in those who are not candidates for trial branched or fenestrated stent graft devices. Due to significant motion in the aortic arch and its interaction with unreinforced in situ fenestrations, questions remain about the durability of the procedure. Most endovascular interventionalists using this procedure have currently relegated it to a bail out maneuver for unintended vessel coverage or for urgent/emergent situations where parallel grafts are deemed less suitable.

\section{Back table modification}

Back table modification of commercially available thoracic endografts to create fenestrations or side branches is the least common employed endovascular technique in the management of aortic arch pathology in high risk patients. PMEGs originally were used in the management of juxtarenal, pararenal or thoracoabdominal aortic aneurysms with reasonable success in patients deemed unfit for open repair (27). Reyes Valdivia et al. (28) recently published a systematic review of PMEGs in the treatment of aortic arch diseases and found just six publications including 239 patients. Most patients (70\%) came from just four high volume centers and involved a single home-made 


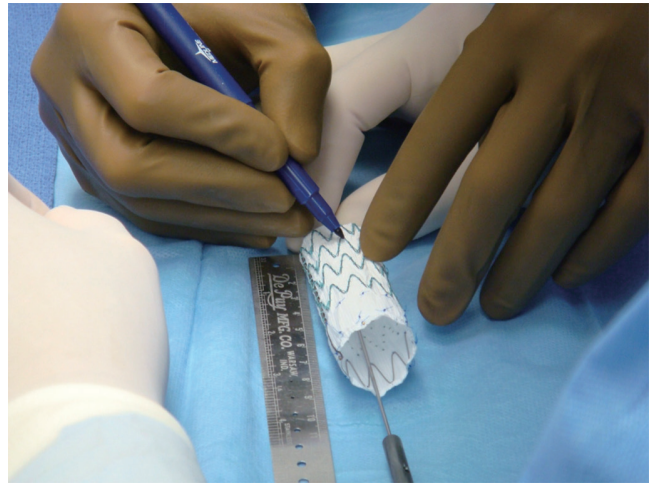

Figure 6 Marking PMEG device. Based upon the patient preoperative CT scan, sites for custom fenestrations are marked on the TEVAR graft. PMEG, physician modified endograft; TEVAR, thoracic endovascular aneurysm repair.

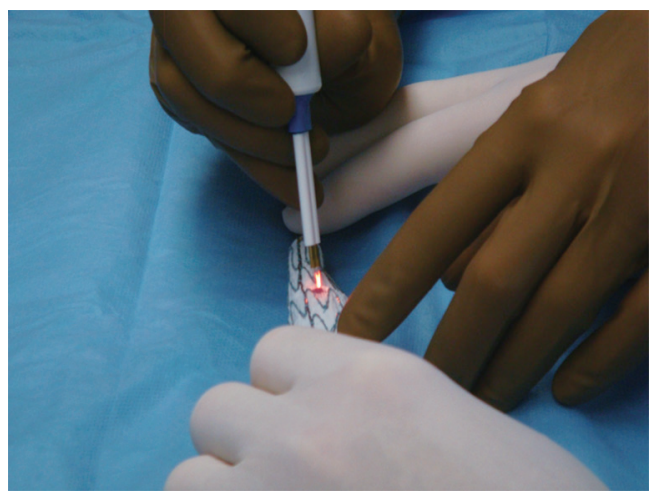

Figure 7 Creating fenestrations of PMEG. Ophthalmologic cautery is used to create fenestrations in the fabric. The fenestrations are reinforced with a locking suture around a radiopaque marker. PMEG, physician modified endograft.

fenestration in Zone 2 for the left subclavian artery. Technical success was high at $98.3 \%$. Thirty-day mortality rate was low at $2.9 \%$ with a stroke rate reported of $2.1 \%$.

The role of back table modifications of a thoracic aortic endograft for use in the abdominal aorta, much less the aortic arch, remains largely unclear. The Society for Vascular Surgery (SVS) issued an advisory statement in 2013 regarding physician modified devices and noted that both local institutional review board (IRB) and FDA Investigator Device Exemption (IDE) approvals should be obtained prior to use of a PMEG device (29). The IDE process includes creating a research study protocol, case reporting forms, and submission of an IDE request to the FDA. A study coordinator and monitor are required to be part of the study to ensure the informed consent process, capture of all study data per the protocol and the timely reporting of adverse event data to the IRB and to the FDA. Yearly study reports to the IRB and FDA are standard, as well as audits of the data and case reporting. The purpose of the IDE is to capture data similar to a clinical trial. By enrolling patients and capturing data as part of an IDE, the procedures are typically eligible for coverage by Medicare and reimbursement.

\section{Technique}

Back table modification of thoracic endografts involves either partial or complete unsheathing of the TEVAR device. Utilizing a pre-printed template, the locations of the intended fenestrations are marked on the graft (Figure 6). Utilizing an eye cautery device, fenestrations are created in the TEVAR graft (Figure 7). A 5-0 polyester locking suture (Ethibond, Ethicon) is used to reinforce each fenestration. Radiopaque markers are sutured around the fenestration using coronary graft markers or gold beads (Accellent, Wilmington, MA, USA). Most physicians creating back table fenestrations use a Cook TX2 polyester graft (Cook Medical, Bloomington, IN, USA) or a Medtronic Talent polyester graft (Medtronic Vascular, Santa Rosa, CA, USA) rather than a PTFE graft. The Cook TX2 device, with its trigger wires that keep the graft attached to the delivery system at the top and bottom of the endograft, allows for the creation of diameter reducing ties. This technique involves completely unsheathing the TEVAR device and removing a section of the polyurethane that houses the trigger wires distally. A trigger wire is then retrieved and then rerouted in and out of the fabric using a long spinal needle. This is performed on the opposite side of the graft from the fenestrations. Next, utilizing prolene sutures, loops are created to bring together multiple stent rings on the graft. The prolene is looped around the trigger wire before it is tied. This maneuver will partially restrain the device on the posterior side of the graft to the fenestrations and will allow for catheter manipulation within the graft. Each fenestration is then cannulated, as well as the intended branch vessel. Once sheaths have been advanced through the fenestrations and into the branch vessel, the trigger wires are removed, the loops come undone and the TEVAR device fully expands. Authors have also described pre-cannulating the fenestrations with 0.014 wires and snaring these wires and bringing them out through the intended target vessel.

Once the fenestrations have been created, the device is reloaded back into its sheath or a larger Check-Flo Sheath 


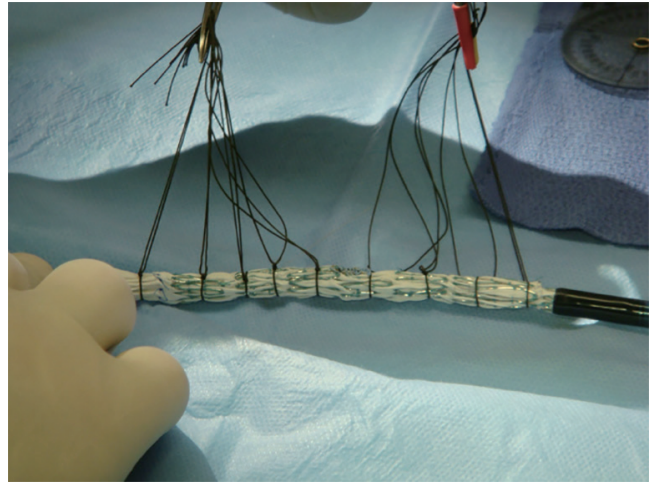

Figure 8 Resheathing PMEG device. Following creation of the fenestrations, the device is carefully placed back in the delivery sheath utilizing restraining ties and a freer elevator. PMEG, physician modified endograft.

(Cook Medical, Bloomington, IN, USA) using silk ties around the stent frame and manual force to reload the device (Figure 8). Care must be taken not to injure the delivery sheath before it goes through the patient's iliac vessels.

PMEGs in the aortic arch have the least amount of supporting data. Concerns about quality and long-term structural integrity of homemade fenestrations or branches in the setting of a mobile aortic arch have limited its widespread application in high risk patients. Some authors have reported the use of 3-D printed models of the patient's anatomy or printing computer aided design templates to guide the location of fenestrations or branches on the TEVAR device (30). There is considerable time needed to partially or completely unsheathe a TEVAR device and create fenestrations or branches, sew radiopaque markers, and reinforce the fenestrations with suture. In patients presenting with rupture and or hemodynamic instability there is typically not enough time to employ this endovascular technique.

\section{Conclusions}

Parallel and PMEGs are infrequently used in most centers except in extenuating circumstances of either high-risk patients who are not candidates for branched or fenestrated trial devices or as a bail out maneuver during complicated TEVAR. We, and others, have avoided PMEGs due to the inconsistent results and medicolegal risk associated with altering a medical device. We have found parallel grafts as a technically easier option in such extenuating circumstances. Physicians treating patients with complex aortic arch aneurysm and dissections should have a clear understanding of the available endovascular techniques for emergent situations as described above. The use of parallel grafts or PMEGs may be the only option for repair in certain patients or in emergent situations. The high technical success and early results justify their use in such situations, however the risk for Type 1 or 3 endoleak is not insignificant and reintervention is frequently needed. In the next several years, multiple branched and fenestrated endovascular thoracic stent grafts for use in the aortic arch will hopefully be commercially approved and obviate the need for parallel and physician modified devices.

\section{Acknowledgments}

Funding: None.

\section{Footnote}

Conflicts of Interest: The authors have no conflicts of interest to declare.

Open Access Statement: This is an Open Access article distributed in accordance with the Creative Commons Attribution-NonCommercial-NoDerivs 4.0 International License (CC BY-NC-ND 4.0), which permits the noncommercial replication and distribution of the article with the strict proviso that no changes or edits are made and the original work is properly cited (including links to both the formal publication through the relevant DOI and the license). See: https://creativecommons.org/licenses/by-nc-nd/4.0/.

\section{References}

1. De Bakey ME, Crawford ES, Cooley DA, et al. Successful resection of fusiform aneurysm of aortic arch with replacement by homograft. Surg Gynecol Obstet 1957;105:657-64.

2. Svensson LG. Antegrade perfusion during suspended animation? J Thorac Cardiovasc Surg 2002;124:1068-70.

3. Shah PJ, Estrera AL, Miller CC 3rd, et al. Analysis of ascending and transverse aortic arch repair in octogenarians. Ann Thorac Surg 2008;86:774-9.

4. Bachet J. Open repair techniques in the aortic arch are still superior. Ann Cardiothorac Surg 2018;7:328-44.

5. Chakos A, Jbara D, Yan TD, et al. Long-term survival and related outcomes for hybrid versus traditional arch repair-a meta-analysis. Ann Cardiothorac Surg 2018;7:319-27. 
6. Hanif H, Dubois L, Ouzounian M, et al. Aortic Arch Reconstructive Surgery With Conventional Techniques vs Frozen Elephant Trunk: A Systematic Review and MetaAnalysis. Can J Cardiol 2018;34:262-73.

7. Hori D, Okamura H, Yamamoto T, et al. Early and midterm outcomes of endovascular and open surgical repair of non-dissected aortic arch aneurysm $†$. Interact Cardiovasc Thorac Surg 2017;24:944-50.

8. Khullar V, Schaff HV, Dearani JA, et al. Open Surgical Repair Remains the Gold Standard for Treating Aortic Arch Pathology. Ann Thorac Surg 2017;103:1413-20.

9. Tanaka Y, Mikamo A, Suzuki R, et al. Mortality and morbidity after total aortic arch replacement. Ann Thorac Surg 2014;97:1569-75.

10. Bavaria J, Vallabhajosyula P, Moeller P, et al. Hybrid approaches in the treatment of aortic arch aneurysms: postoperative and midterm outcomes. J Thorac Cardiovasc Surg 2013;145:S85-90.

11. Elhelali A, Hynes N, Devane D, et al. Hybrid repair versus conventional open repair for thoracic aortic arch aneurysms. Cochrane Database Syst Rev 2021;6:CD012923.

12. Yan TD, Tian DH, LeMaire SA, et al. Standardizing clinical end points in aortic arch surgery: a consensus statement from the International Aortic Arch Surgery Study Group. Circulation 2014;129:1610-6.

13. Mangialardi N, Serrao E, Kasemi H, et al. Chimney technique for aortic arch pathologies: an 11-year singlecenter experience. J Endovasc Ther 2014;21:312-23.

14. Lindblad B, Bin Jabr A, Holst J, et al. Chimney Grafts in Aortic Stent Grafting: Hazardous or Useful Technique? Systematic Review of Current Data. Eur J Vasc Endovasc Surg 2015;50:722-31.

15. Voskresensky I, Scali ST, Feezor RJ, et al. Outcomes of thoracic endovascular aortic repair using aortic arch chimney stents in high-risk patients. J Vasc Surg 2017;66:9-20.e3.

16. McWilliams RG, Murphy M, Hartley D, et al. In situ stent-graft fenestration to preserve the left subclavian artery. J Endovasc Ther 2004;11:170-4.

17. Murphy EH, Dimaio JM, Dean W, et al. Endovascular repair of acute traumatic thoracic aortic transection with laser-assisted in-situ fenestration of a stent-graft covering the left subclavian artery. J Endovasc Ther 2009;16:457-63.

18. Ahanchi SS, Almaroof B, Stout CL, et al. In situ laser fenestration for revascularization of the left subclavian artery during emergent thoracic endovascular aortic repair. J Endovasc Ther 2012;19:226-30.
19. Redlinger RE Jr, Ahanchi SS, Panneton JM. In situ laser fenestration during emergent thoracic endovascular aortic repair is an effective method for left subclavian artery revascularization. J Vasc Surg 2013;58:1171-7.

20. Guidoin R, Lin J, Nutley M, et al. Commentary: Honing the Technique of In Situ Stent-Graft Fenestration. J Endovasc Ther 2021;28:53-5.

21. Riga CV, Bicknell CD, Basra M, et al. In vitro fenestration of aortic stent-grafts: implications of puncture methods for in situ fenestration durability. J Endovasc Ther 2013;20:536-43.

22. Longo GM, Pipinos II. Endovascular techniques for arch vessel reconstruction. J Vasc Surg 2010;52:77S-81S.

23. Zeng Q, Zhou X, He Y, et al. Experimental Analysis of In Situ Fenestration of Endovascular Stent-Grafts: Comparison between Needle and Laser Puncture. Ann Vasc Surg 2021;77:280-7.

24. Li DL, Zeng QL, Xiang YL, et al. Experimental Analysis of the Quality of Needle-Assisted Fenestration in Aortic Stent-Grafts and the Differences Between Gradual and Rapid Balloon Dilation. J Endovasc Ther 2021;28:44-52.

25. Crawford SA, Sanford RM, Forbes TL, et al. Clinical outcomes and material properties of in situ fenestration of endovascular stent grafts. J Vasc Surg 2016;64:244-50.

26. Kopp R, Katada Y, Kondo S, et al. Multicenter Analysis of Endovascular Aortic Arch In Situ Stent-Graft Fenestrations for Aortic Arch Pathologies. Ann Vasc Surg 2019;59:36-47.

27. Canonge J, Jayet J, Heim F, et al. Comprehensive Review of Physician Modified Aortic Stent Grafts: Technical and Clinical Outcomes. Eur J Vasc Endovasc Surg 2021;61:560-9.

28. Reyes Valdivia A, Pitoulias G, Pitoulias A, et al. Systematic Review on the Use of Physician-Modified Endografts for the Treatment of Aortic Arch Diseases. Ann Vasc Surg 2020;69:418-25.

29. White RA. Advisory statement on clinical use of modified aortic endografts from the Society for Vascular Surgery®. J Vasc Surg 2013;57:832-3.

30. Rynio P, Kazimierczak A, Jedrzejczak T, et al. A 3-Dimensional Printed Aortic Arch Template to Facilitate the Creation of Physician-Modified Stent-Grafts. J Endovasc Ther 2018;25:554-8.

Cite this article as: Atkins MD, Lumsden AB. Parallel grafts and physician modified endografts for endovascular repair of the aortic arch. Ann Cardiothorac Surg 2022;11(1):16-25. doi: 10.21037/acs-2021-taes-171 